\title{
GAUSS FORMULAS FOR THE DIRICHLET PROBLEM
}

BY A. H. STROUD AND DAVID L. BARROW

Communicated by Alston S. Householder, May 13, 1974

The 2-dimensional Dirichlet problem for Laplace's equation is the problem of finding $u(x, y)$ to satisfy

$$
\frac{\partial^{2} u}{\partial x^{2}}+\frac{\partial^{2} u}{\partial y^{2}}=0 \quad \text { in } R, \quad u(x, y) \text { given on } B \text {. }
$$

Here $R$ is an open, bounded, simply-connected region in the $(x, y)$-plane and $B$ is the boundary of $R$. We assume that $B$ is a rectifiable Jordan curve.

It is well known (see, for example, Protter and Weinberger [4, p. 85]) that the solution of $(1)$ at a point $\left(x_{*}, y_{*}\right)$ in $R$ can be written

$$
u\left(x_{*}, y_{*}\right)=\int_{B} w\left(x, y ; x_{*}, y_{*}\right) u(x, y) d s,
$$

where

$$
w\left(x, y ; x_{*}, y_{*}\right) \equiv-\frac{\partial G}{\partial n}\left(x, y ; x_{*}, y_{*}\right)
$$

is the normal derivative of Green's function for $R$.

We are interested in approximations of the form

$$
u\left(x_{*}, y_{*}\right) \simeq \sum_{k=1}^{N} A_{k} u\left(x_{k}, y_{k}\right)
$$

where the $A_{k}$ are real constants and the $\left(x_{k}, y_{k}\right)$ are points on $B$. We say that (3) is a harmonic interpolation formula of degree $d$ if (3) is an equality for all harmonic polynomials of degree $\leqslant d$, and if there is at least one harmonic polynomial of degree $d+1$ for which (3) is not an equality. A harmonic polynomial of degree $d$ is any linear combination of the linearly independent polynomials

AMS (MOS) subject classifications (1970). Primary 41 A55, 41 A30; Secondary 65 N35. 
1, $\operatorname{Re} z^{m}, \operatorname{Im} z^{m}, \quad m=1, \cdots, d, \quad z=x+i y$.

In view of equation (2) we can alternately consider an approximation (3) to be a numerical quadrature formula.

For a given $N$ we wish to find $\left(x_{k}, y_{k}\right), A_{k}, k=1, \cdots, N$, with all the $A_{k}>0$ so that (3) will be a harmonic interpolation formula of as high degree as possible. Since each $\left(x_{k}, y_{k}\right)$ is restricted to lie on $B$, there are $2 N$ free parameters in the choice of the $\left(x_{k}, y_{k}\right), A_{k}$. Since there are $2 N$ -1 linearly independent harmonic polynomials of degree $\leqslant N-1$, we would expect that by fixing one of the parameters in advance, say $\left(x_{N}, y_{N}\right)$, we could uniquely determine the other parameters so that (3) has degree $N$ 1. We call such a formula a Gauss harmonic interpolation formula or, for short, a Gauss formula.

The only region $R$ for which Gauss formulas are known to exist for all $N$ is the circle. For the unit circle the harmonic polynomials (4) are 1, $\cos (m \theta), \sin (m \theta), m=1, \cdots, d$, where $x=\cos \theta, y=\sin \theta$. These polynomials, considered as functions of one variable defined on $x^{2}+y^{2}=1$, form a periodic Tchebycheff system, and the existence of an $N$-point Gauss formula follows from the theory of Tchebycheff systems (see Karlin and Studden $[2, \S \S 2.3,6.2])$.

For an arbitrary region $R$, Stroud, Chen, Wang, and Mao [5] show the existence of Gauss formulas of degree 2 with $N=3$. It is not difficult to show that if $\left(x_{1}, y_{1}\right)$ and $\left(x_{2}, y_{2}\right)$ are the endpoints of a straight line segment which contains $\left(x_{*}, y_{*}\right)$, then $\left(x_{1}, y_{1}\right),\left(x_{2}, y_{2}\right)$ give a Gauss formula of degree 1 .

Our main result is

Theorem 1. Assume $B$ is a rectifiable Jordan curve, $\left(x_{N}, y_{N}\right)$ is any given point on $B,\left(x_{*}, y_{*}\right)$ is any given point in $R$, and $N \geqslant 2$. Then the $N$-point Gauss formula exists.

We say that $R$ is strictly convex if $R$ is convex and if $B$ contains no straight line segments. We have also proven

THEOREM 2. If $R$ is not strictly convex, then the harmonic polynomials (4), considered as functions of one variable defined on B, are not a Tchebycheff system.

From Theorem 2 it follows that, at least for some regions $R$, the theory of Tchebycheff systems will not suffice to prove Theorem 1. Proofs of these 
theorems will be given in [1]. Let $R$ be a continuous mapping from an open subset $D$ of Euclidean $n$-space $E^{n}$ into $E^{n}$. The proof of Theorem 1 is based on the concept of the degree of $F$, at a point $\phi$ in $E^{n}$, with respect to an open subset $C$ of $D$. For the definition and properties of the degree of a mapping see, for example, Ortega and Rheinboldt [3, pp. 148-161].

We believe Theorem 1 is important because it gives what seems to be basic new information about harmonic polynomials. Also the method used to prove Theorem 1 has not previously been used to show the existence of a class of Gauss quadrature formulas. In fact, we do not know the proof of existence of any other class of Gauss quadrature formulas when the set of basis functions are not a Tchebycheff system.

\section{REFERENCES}

1. David L. Barrow and A. H. Stroud, Existence of Gauss harmonic interpolation formulas (submitted for publication).

2. S. Karlin and W. J. Studden, Tchebycheff systems: With applications in analysis and statistics, Pure and Appl. Math., vol. 15, Interscience, New York, 1966. MR 34 \#4757.

3. J. M. Ortega and W. C. Rheinboldt, Iterative solution of nonlinear equations in several variables, Academic Press, New York, 1970. MR $42 \# 8686$.

4. M. H. Protter and H. F. Weinberger, Maximum principles in differential equations, Prentice-Hall, Englewood Cliffs, N. J., 1967. MR 36 \#2935.

5. A. H. Stroud, K.-W. Chen, P.-L. Wang and Z. Mao, Some second and third degree harmonic interpolation formulas, SIAM J. Numer. Anal. 8 (1971), 681-692. MR 45 \#7928.

DEPARTMENT OF MATHEMATICS, TEXAS A \& M UNIVERSITY, COLLEGE STATION, TEXAS $\mathbf{7 7 8 4 3}$ 